\title{
Robust Fault Detection of Non-linear Systems using Set-membership State Estimation based on Constraint Satisfaction
}

\author{
Sebastian Tornil-Sin ${ }^{\mathrm{a}}$, Carlos Ocampo-Martinez ${ }^{\mathrm{a}, \mathrm{b}}$, Vicenç Puiga, ${ }^{\mathrm{a}, \mathrm{b}}$, Teresa Escobet ${ }^{\mathrm{a}}$ \\ ${ }^{a}$ Advanced Control Systems Research Group \\ Universitat Politècnica de Catalunya (UPC) \\ Rambla de Sant Nebridi, 10, 08222 Terrassa (Spain) \\ \{sebastian.tornil, teresa.escobet\} @upc.edu \\ ${ }^{b}$ Institut de Robòtica i Informàtica Industrial, CSIC-UPC \\ C/. Llorens i Artigas, 4-6, 08028 Barcelona (Spain) \\ \{cocampo, vpuig\} @iri.upc.edu
}

\begin{abstract}
In this paper, the robust fault detection problem for non-linear systems considering both bounded parametric modelling errors and measurement noises is addressed. The non-linear system is monitored by using a state estimator with bounded modelling uncertainty and bounded process and measurement noises. Additionally, time-variant and time-invariant system models are taken into account. Fault detection is formulated as a set-membership state estimation problem, which is implemented by means of constraint satisfaction techniques. Two solutions are presented: the first one solves the general case while the second solves the time-variant case, being this latter a relaxed solution of the first one. The performance of the time-variant approach is tested in two applications: the well-known quadruple-tank benchmark and the dynamic model of a representative portion of the Barcelona's sewer network. In both applications, different scenarios are presented: a faultless situation and some faulty situations. All considered scenarios are intended to show the effectiveness of the presented approach.
\end{abstract}

Keywords: Fault detection, robustness, intervals, set-membership estimation, constraints satisfaction.

\section{Introduction}

Model-based fault detection methods rely on the concept of analytical redundancy. The simplest analytical redundancy scheme consists in the comparison of system output measurements with the corresponding analytically computed values, obtained from measurements of other variables and/or from previous measurements of the same variable by means of a model. In the general case, different estimations of a same variable, measured or not, can be compared. The resultant differences are called residuals, which are indicative of faults in the system. Under ideal conditions, residuals are zero in the absence of faults and non-zero when a fault is present. However, modelling errors, disturbances and noises in complex engineering systems are inevitable, and hence appears the necessity of developing robust fault detection algorithms. The robustness of a fault detection system indicates its ability to distinguish between faults and model-reality differences [5].

Classical approaches facing disturbances and modelling errors employ the disturbance decoupling principle for obtaining a residual sensitive to faults but not to these errors. Techniques such as unknown input observers [6], eigenstructure assignment [5] or structured parity equations [8], among others, can be found in the literature. On the other hand, process and measurement noises are usually stochastically modelled (the typical assumption is a zero mean white noise) and their effect is considered by using statistical decision methods [2].
However, such approaches present several drawbacks. First, decoupling from modelling errors (specially for non-linear models) is difficult to solve since the distribution matrix is normally unknown, time varying and therefore should be estimated. Moreover, the number of decoupled disturbances/modelling errors is limited by the degree of freedom in the residual generation procedure [8]. As an alternative strategy, disturbances/model errors are assumed to be bounded and its effect is propagated to the residual using, for example, interval methods [19]. Second, in many practical situations it is not realistic to assume a probability distribution for the noise, being more natural to assume that only bounds on the noise signals are available. In this case, the so called set-membership approach [16] can be used in the context of fault detection as suggested in [24]. In both cases, the advantage of the bounded description of uncertainty is that it does not require restrictive assumptions (e.g., a small number of unknown disturbances/parameters, a known probability distribution, etc.). However, a limitation is that faults which produce a residual deviation smaller than the residual uncertainty due to model uncertainty will be missed (missed alarms).

In this paper, the robust fault detection problem for nonlinear systems considering both bounded parametric modelling errors and measurement noises is addressed. Fault detection is formulated as a set-membership estimation problem, being this fact one of the main contributions. A state estimator that describes the set of all the states consistent with modelling uncertainty, measured data and noise bounds is presented. Several 
works such as in [3], [11], [14] and [22], among others, have addressed this issue. Unfortunately, the set obtained through this way might become extremely complex due to the non-linear nature of the model [14]. In [24], a state estimator based on enclosing the set of states by the smallest ellipsoid is proposed following the algorithms proposed by [15]. However, in this approach only additive uncertainty is considered, but not the multiplicative uncertainty introduced by unknown parameters. The implementation of the state estimator based on constraint satisfaction techniques discussed in [12] is presented. The obtained fault detection algorithm can be considered as an improvement of the approach to robust fault detection proposed by [21], which only considers system trajectories obtained from the uncertain parameter interval vertices, assuming that the monotonicity property holds.

The paper is organized as follows: In Section 2, the problem of fault detection for non-linear time-varying systems using set-membership estimation is presented. Section 3 reviews the constraint satisfaction background. Section 4 addresses the implementation of set-membership state estimation in fault detection using constraints satisfaction. Section 5 and 6 illustrate the performance of the proposed approach using two case studies: the well-known multivariable control benchmark based on the four tank system and a representative piece of the Barcelona's sewer network. Finally, Section 7 summarizes the main conclusions of the paper.

\section{Notation}

In the sequel, for a given variable $x$, its minimum value is denoted as $\underline{x}$ while its maximum value is denoted as $\bar{x}$. Moreover, $\mathbb{I}^{n}$ is the set of all interval vectors (boxes) in $\mathbb{R}^{n}$.

\section{Set-Membership State Estimation applied to Fault De- tection}

\subsection{System model}

Consider the following discrete-time non-linear dynamical model describing the behaviour of the system to be monitored:

$$
\begin{aligned}
x_{k+1} & =g\left(x_{k}, u_{k}, \theta_{k}, w_{k}\right), \\
y_{k} & =h\left(x_{k}, u_{k}, \theta_{k}\right)+v_{k},
\end{aligned}
$$

where:

- $x \in \mathbb{X} \subseteq \mathbb{R}^{n}$ is the vector of system states, $u \in \mathbb{R}^{m}$ is the vector of system inputs and $y \in \mathbb{R}^{p}$ is the vector of system outputs.

- $w_{k} \in \mathbb{R}^{n}$ and $v_{k} \in \mathbb{R}^{p}$ are process and measurement noises, which are considered unknown but bounded, i.e., $v_{k} \in \mathbb{V}$ and $w_{k} \in \mathbb{W}$, where $\mathbb{V}$ and $\mathbb{W}$ are the interval boxes

$$
\begin{aligned}
\mathbb{V} & =\left\{v \in \mathbb{R}^{p} \mid \underline{v} \leq v \leq \bar{v}\right\}, \\
\mathbb{W} & =\left\{w \in \mathbb{R}^{n} \mid \underline{w} \leq w \leq \bar{w}\right\} .
\end{aligned}
$$

- $g: \mathbb{R}^{n} \mapsto \mathbb{R}^{n}$ and $h: \mathbb{R}^{n} \mapsto \mathbb{R}^{p}$ are the state-space and measurement non-linear functions, respectively.
- $\mathbb{X}_{0}$ describes the set of initial states as

$$
\mathbb{X}_{0}=\left\{x \in \mathbb{R}^{n} \mid \underline{x}_{0} \leq x \leq \bar{x}_{0}\right\}
$$

- $\theta_{k} \in \mathbb{R}^{q}$ is a vector of uncertain parameters with their values bounded by a compact set $\theta_{k} \in \Theta$ of box type

$$
\Theta=\left\{\theta \in \mathbb{R}^{q} \mid \underline{\theta} \leq \theta \leq \bar{\theta}\right\} .
$$

Parameter uncertainty comes from physical modelling or from the set-membership parameter estimation algorithms applied in a non-faulty situation [16]. Depending of the knowledge about parameter variance, the following additional equation can be added to the system description (1a)-(1b):

$$
\theta_{k+1}=\theta_{k}+p_{k}
$$

with $p_{k} \in \mathbb{P}$, where $\mathbb{P}$ is an interval box

$$
\mathbb{P}=\left\{p \in \mathbb{R}^{q} \mid \underline{p} \leq p \leq \bar{p}\right\}
$$

Here, two different cases can be considered:

- time-invariant case, what implies $p_{k}=0$, and

- time-varying case, what implies $p_{k} \in \mathbb{P}$.

In the first case, the parameter is unknown within $\Theta$ but it is known that it will not vary. In the second case, the parameter variation is specifically bounded by the interval box $\mathbb{P}$.

The first case might represent situations where an initial variance comes from component specifications that are known only with a mean and variance in the beginning of the fault detection task. The second case might represent a system that has been identified over a number of operation conditions, each with a different $\theta \in \Theta$, but with the inter-sample variance bounded by $\mathbb{P}$.

\subsection{Fault detection using set-membership estimation}

Let $\tilde{u_{k}}$ and $\tilde{y_{k}}$ denote respectively the sequence of measured input/output variables from the initial time to the time instant $k$, i.e., $\left\{u_{j}\right\}_{j=0}^{k}$ and $\left\{y_{j}\right\}_{j=0}^{k}$. Also, $\tilde{w}_{k}, \tilde{v_{k}}, \tilde{\theta_{k}}$ and $\tilde{p_{k}}$ denote a sequence of unknown but bounded (inside their respective sets) process and measurement noises, parameter uncertainty and parameter variations, that is, $\left\{w_{j}\right\}_{j=0}^{k},\left\{v_{j}\right\}_{j=0}^{k},\left\{\theta_{j}\right\}_{j=0}^{k}$ and $\left\{p_{j}\right\}_{j=0}^{k}$. When time-invariant systems are considered, then $\tilde{p_{k}}=\{0\}$.

Definition 2.1 (Set-membership state estimation). Given the system described by (1a)-1b, an initial compact set $\mathbb{X}_{0}$ and a sequence of measured inputs $\tilde{u_{k}}$ and outputs $\tilde{y}_{k}$ taken from the real system, the set of estimated states at time $\mathrm{k}$ using the setmembership approach is expressed as

$$
\begin{aligned}
\mathbb{X}_{k}= & \left\{x_{k} \mid \exists \tilde{w_{k}}, \tilde{v_{k}}, \tilde{\theta_{k}}, \tilde{p_{k}}, x_{0} \in \mathbb{X}_{0},\right. \\
& \left(x_{j}=g\left(x_{j-1}, u_{j-1}, \theta_{j-1}, w_{j-1}\right)\right)_{j=1}^{k}, \\
& \left(y_{j}=h\left(x_{j}, u_{j}, \theta_{j}\right)+v_{j}\right)_{j=0}^{k}, \\
& \left.\left(\theta_{j}=\theta_{j-1}+p_{j-1}\right)_{j=0}^{k}\right\}
\end{aligned}
$$


Definition 2.2 (Fault detection). Given a sequence of measured inputs $\tilde{u_{k}}$ and outputs $\tilde{y_{k}}$ of the real system, a fault is said to be detected at time $\mathrm{k}$ if there does not exist a set of sequences $\tilde{v_{k}}$, $\tilde{w}_{k}, \tilde{\theta_{k}}$ and $\tilde{p_{k}}$ which satisfy the nominal system (la) with initial condition $\mathbb{X}_{0}$ and noise, disturbances and parameters that belongs to $\mathbb{V}, \mathbb{W}, \Theta$ and $\mathbb{P}$, respectively.

According to Definition 2.2, a fault can be detected using a set-membership estimator when the set of estimated states in Definition 2.1 is the empty, i.e., $\mathbb{X}_{k}=\emptyset$.

\section{Constraints Satisfaction Background}

Fault detection based on set-membership state estimate presented in Definition 2.2 can be naturally handled as Constraint Satisfaction Problem (CSP). Thus, the definition of a CSP is introduced and background related to this approach is presented and discussed. Then, the proposed approach is applied to the fault detection problem.

\subsection{Constraints Satisfaction Problem}

A CSP on sets can be formulated as a 3-tuple $\mathbb{H}=(\mathbb{Z}, \mathbb{D}, \mathbb{C})$ [12], where

- $\mathbb{Z}=\left\{z_{1}, \cdots, z_{n}\right\}$ is a finite set of variables,

- $\mathbb{D}=\left\{\mathbb{D}_{1}, \cdots, \mathbb{D}_{n}\right\}$ is the set of their domains represented by closed sets, and

- $\mathbb{C}=\left\{c_{1}, \cdots, c_{m}\right\}$ is a finite set of constraints relating variables of $\mathbb{Z}$.

A solution point of $\mathbb{H}$ is an $n$-tuple $\left(\tilde{z}_{1}, \cdots, \tilde{z}_{n}\right) \in \mathbb{D}$ such that all constraints in $\mathbb{C}$ are satisfied. The set of all solution points of $\mathbb{H}$ is denoted by $\mathbb{S}(\mathbb{H})$. This set is called the global solution set. The variable $z_{i} \in \mathbb{Z}$ is consistent in $\mathbb{H}$ if and only if $\forall \tilde{z}_{i} \in \mathbb{D}_{i}$

$$
\exists\left(\tilde{z}_{1} \in \mathbb{D}_{1}, \cdots, \tilde{z}_{i-1} \in \mathbb{D}_{i-1}, \tilde{z}_{i+1} \in \mathbb{D}_{i+1}, \cdots, \tilde{z}_{n} \in \mathbb{D}_{n}\right)
$$

such as $\left(\tilde{z}_{1}, \cdots, \tilde{z}_{n}\right) \in \mathbb{S}(\mathbb{H})$.

The solution of a CSP is said to be globally consistent if and only if every variable is consistent. A variable is locally consistent if and only if it is consistent with respect to all directly connected constraints. Thus, the solution of the CSP is said to be locally consistent if all variables are locally consistent. An algorithm for finding an approximation of the solution set of a CSP can be found in [12].

It is well known that the solution of CSPs involving sets has a high complexity [12]. A first relaxation consists in approximating the variable domains by means of intervals and finding the solution through solving an Interval Constraints Satisfaction Problem (ICSP) [10]. The determination of the intervals that approximate in a more fitted form the sets that define the variable domains requires global consistency, what demands a high computational cost [10]. A second relaxation consists in solving the ICSP by means of local consistency techniques, deriving on conservative intervals. Interval constraint satisfaction algorithms have polynomial-time worst case complexity since they implement local reasoning on constraints to remove inconsistent values from variable domains [12].

\subsection{Real Paver}

In this paper, the CSP problem derived from the set-membership state estimation problem is relaxed to an ICSP problem that is solved using a tool based on interval constraint propagation, known as Real Paver. This tool has been designed and developed by research team of Professor Granvilliers [9]. The goal of this software is to determine the solution of an ICSP in the case that its domains are represented by closed real intervals. The approach consists in iterating two main operations: domain contraction and propagation, which can be combined with an additional operation known as bisection, if necessary. The solution provides refined interval domains consistent with the set of ICSP constraints.

\subsubsection{Contraction}

A contractor is an operator that reduces domains. Applied to the solution $\mathbb{H}$ of a CSP, an operator $\mathbb{C}_{\mathbb{H}}: \mathbb{I R}^{n} \rightarrow \mathbb{I}^{n}$ is a contractor if it satisfies

$$
\forall[z] \in \mathbb{D}:\left\{\begin{array}{l}
\mathbb{C}_{\mathbb{H}}([z]) \subset[z] \\
\mathbb{C}_{\mathbb{H}}([z]) \cap \mathbb{S}(\mathbb{H})=[z] \cap \mathbb{S}(\mathbb{H}) .
\end{array}\right.
$$

The purpose of a contractor is to reduce any box $[z]$ without loosing any solution point in $\mathbb{S}(\mathbb{H})$. In [12], a number of contractors for a variety of sets are given. They are algorithms of polynomial complexity that reduce the interval domains of variables which comply with a set of constraints. The application of contractor operators is therefore known as contraction.

\subsubsection{Propagation}

When several constraints are involved, the contractions are sequentially performed until no more significant contraction can be observed. This procedure is known as propagation. The interval propagation method converges to a box which contains all solution vectors of the constraint set. If this box is empty, it means that there is no solution. It can be shown that the box to which the method converges does not depend on the order to which the contractors are applied [12], but the computation time is highly sensitive to this order. There is no optimal order in general, but in practice, one of the most efficient is called forward-backward propagation.

\subsubsection{Bisection}

The combined use of contraction and propagation leads only to obtain a local consistent solution. The locality problem is due to the strategy for reducing domains processes every constraint projection independently. To escape from local consistency, every resultant box from the application of contraction and propagation should be bisected in two sub-boxes, sharing all variable domains of the original box, except the one with largest width, which is split by its mid point. Then, contractors and propagation are applied on the new resultant sub-boxes. This process, called bisection, is iterated until no refinement of those subboxes below to a pre-established amount is achieved. 


\section{Fault Detection using Set-membership State Estimation based on Constraint Satisfaction}

\subsection{Fault detection using CSP: the general case}

The fault detection procedure described in Definition 2.2 requires the computation of the set of estimated states $\mathbb{X}_{k}$ in Definition 2.1. It can be noticed that this problem corresponds naturally to a CSP on sets by introducing the following definitions:

- $\mathbb{Z}=\left\{\widetilde{x}_{k}, \widetilde{w}_{k}, \widetilde{v}_{k}, \widetilde{\theta}_{k}, \widetilde{p}_{k}\right\}$

- $\mathbb{D}=\left\{\widetilde{\mathbb{X}}_{k}, \widetilde{\mathbb{W}}_{k}, \widetilde{\mathbb{V}}_{k}, \widetilde{\Theta}_{k}, \widetilde{\mathbb{P}}_{k}\right\}$, where $\widetilde{\mathbb{X}}_{k}=\left\{\mathbb{X}_{j}\right\}_{j=0}^{k}$, $\widetilde{\mathbb{V}}_{k}=\left\{\mathbb{W}_{j}\right\}_{j=0}^{k}, \widetilde{\mathbb{V}}_{k}=\left\{\mathbb{V}_{j}\right\}_{j=0}^{k}, \widetilde{\Theta}_{k}=\left\{\Theta_{j}\right\}_{j=0}^{k}$ and $\widetilde{\mathbb{P}}_{k}=\left\{\mathbb{P}_{j}\right\}_{j=0}^{k}$, that means the domain of all the variables, and

- $\mathbb{C}=\mathbb{C}_{1} \cup \mathbb{C}_{2} \cup \mathbb{C}_{3}$, where

$$
\begin{aligned}
& \text { - } \mathbb{C}_{1}=\left\{x_{j+1}=f\left(x_{j}, u_{j}, \theta_{j}, w_{j}\right)\right\}_{j=0}^{k}, \\
& \text { - } \mathbb{C}_{2}=\left\{y_{j}=g\left(x_{j}, u_{j}, \theta_{j}\right)+v_{j}\right\}_{j=0}^{k}, \\
& \text { - } \mathbb{C}_{3}=\left\{\theta_{j+1}=\theta_{j}+p_{j}\right\}_{j=0}^{k} .
\end{aligned}
$$

Algorithm 1 allows to solve the set-membership state estimate by solving, at each time iteration, the associated CSP defined above, the process/measurement noise bounds, the set of parameters and the initial state uncertainty.

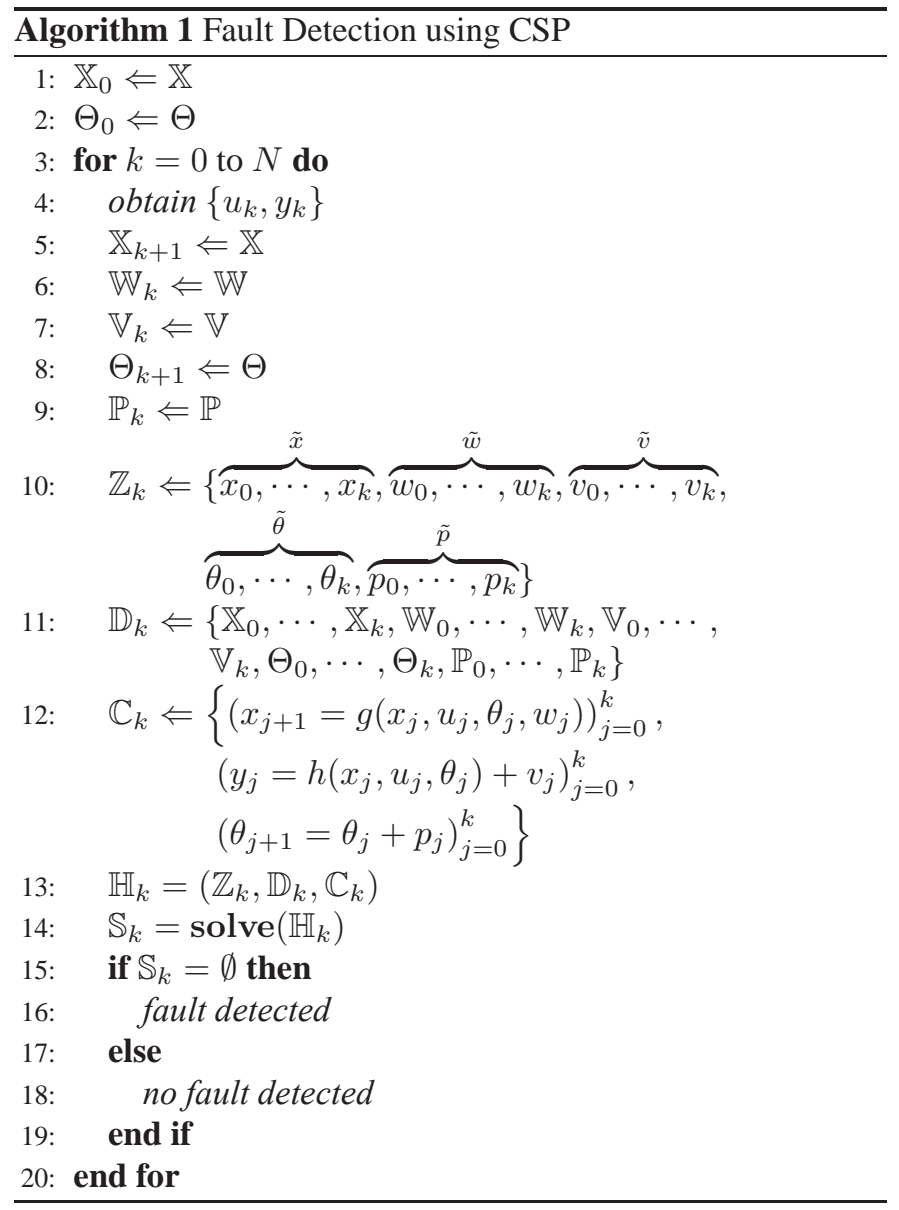

It is well known that the solution of CSP over sets has a high computational complexity. This complexity comes from the fact that to represent the solution sets accurately, they need to be decomposed using subpavings (union of boxes) and to use set computations that involves bisections, which implies exponential growing of the computation times [11].

A possible relaxation in order to reduce computational complexity associated with Algorithm 1 consists in approximating the variable domain sets by means of their interval hulls ${ }^{1}$. Then, this approximation leads to a CSP over intervals, named ICSP [11] and the new set of domains for $\mathbb{H}_{k}=\left(\mathbb{V}_{k}, \mathbb{D}_{k}, \mathbb{C}_{k}\right)$ is expressed as follows ${ }^{2}$ :

$$
\begin{array}{r}
\mathbb{D}_{k}=\left\{\square \mathbb{X}_{0}, \cdots, \square \mathbb{X}_{k}, \square \mathbb{V}_{0}, \cdots, \square \mathbb{V}_{k}\right. \\
\left.\square \mathbb{W}_{0}, \cdots, \square \mathbb{W}_{k}, \square \Theta_{0}, \cdots, \square \Theta_{k}, \square \mathbb{P}_{0}, \cdots, \square \mathbb{P}_{k}\right\}
\end{array}
$$

Moreover, this relaxation allows the use of interval methods, which have efficient operators (contractors) to find the solution of an ICSP [10]. However, the exact interval hull determination of the sets which define the variable domains (i.e., the interval box that approximates in a more fitted form these sets) requires global consistency. This kind of consistency is achieved when all constraints in the ICSP are consistent simultaneously but demands a high computational burden because implies bisections [10]. To further reduce computational complexity, an ICSP can be solved by means of using only contractors. The price is that local consistency is achieved, i.e., every constraint in the ICSP is consistent independently from each other [11]. This leads to the resultant interval hull of the variable domain sets are not the most adjusted but no point solution is lost. Using such relaxations, if the interval hull of the admissible solution set $\mathbb{X}_{k}$ returned by Algorithm 1 is empty, then $\mathbb{X}_{k}$ is an empty set as well and the fault is detected. Otherwise, nothing can be stated since $\square \mathbb{X} \neq \emptyset$ does not imply $\mathbb{X}=\emptyset$.

Another drawback of Algorithm 1 is the increase of computational complexity of the CSP since at each iteration an additional restriction is added. So, the amount of computation burden increases with time, being impossible to operate over a large time interval. This problem can be solved by using a time-sliding window of length $L$ [20].

\subsection{Fault detection using CSP: the time-varying case}

For the case that uncertain parameters are considered to be time-varying (that is, $p_{k} \neq 0$ ), an alternative approach to solve the CSP proposed in Algorithm 1 can be used. This alternative approach consists in admitting the rupture of the existing relations between variables for consecutive time instants given the time-varying assumption of uncertain parameters. This fact makes possible to determinate the interval hull of the set of feasible solutions step by step [7]. In this case, it is only necessary

${ }^{1}$ Giving a variable $z$ and its domain $\mathbb{Z}$, its interval hull is given by

$$
\square \mathbb{Z}_{0}=\left\{z \in \mathbb{R}^{n_{z}} \mid \underline{z}_{0} \leq z \leq \bar{z}_{0}\right\}
$$

${ }^{2}$ Throughout this paper and as notation, the symbol $\square$ (square box) denotes the interval hull of a given set. 
to propagate the set of estimated states $\mathbb{X}_{k}$ from the current iteration to the next.

Summarizing, Algorithm 1 can be approximated as presented in Algorithm 2 under the following considerations:

- a CSP over sets will be relaxed to a CSP over intervals,

- local consistency is used instead of global consistency, and

- domains of variables are computed iteratively from previously computed domains.

However, the problem of uncertainty propagation (wrapping effect) could appear when the CSP is solved in this way. This problem does not appear in isotone systems [7] (see also, monotone systems [1]), which are the systems whose state function is isotone. The monotonicity assumption is also used in the robust fault detection algorithm proposed in [21]. To avoid the wrapping effect due to the approximation of the set of estimated states by its interval hull, a more complex approximation is proposed in [17].

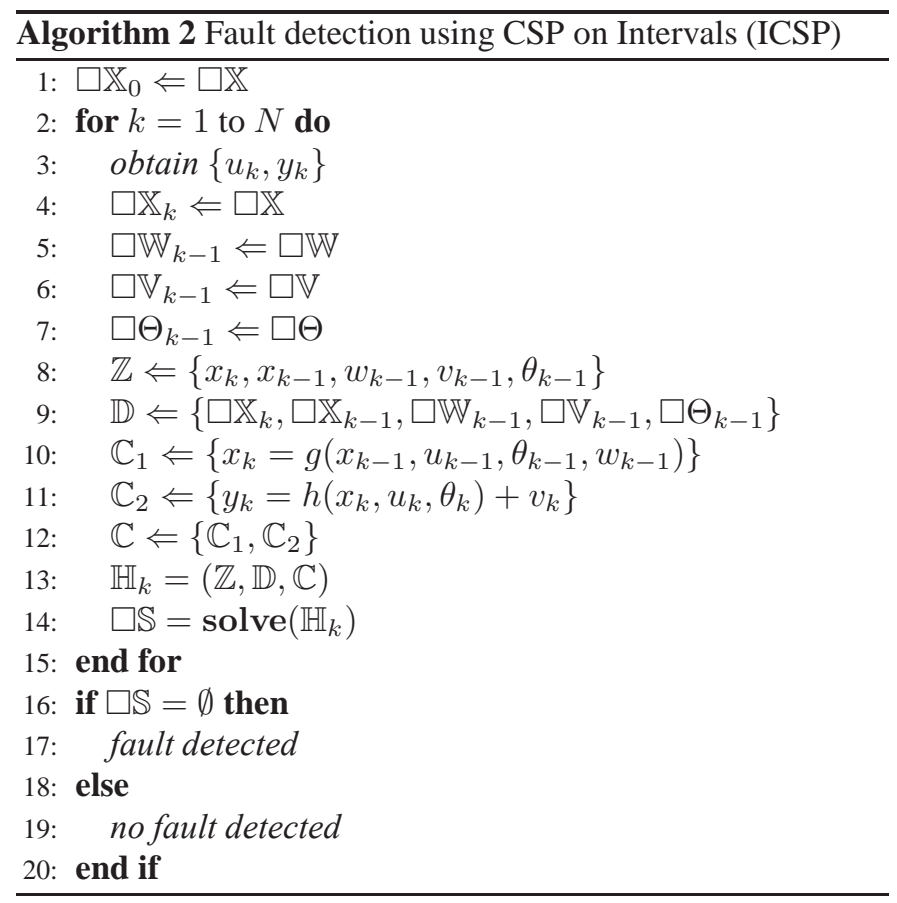

\section{Application Example 1: Quadruple-Tank Process}

As an application example to illustrate the fault detection and state estimation algorithms proposed in this paper, the four tanks system proposed in [13] is used. This is a multivariable laboratory process proposed as a control benchmark.

\subsection{System description}

A schematic diagram of the process is shown in Figure 1. The components of the system are tanks $T_{1}, T_{2}, T_{3}$ and $T_{4}$, bypass valves $V_{1}$ and $V_{2}$, and pumps $P_{1}$ and $P_{2}$. The inputs variables are voltages to the pumps (i.e., $u_{1}=v_{1}$ and $u_{2}=v_{2}$ ).

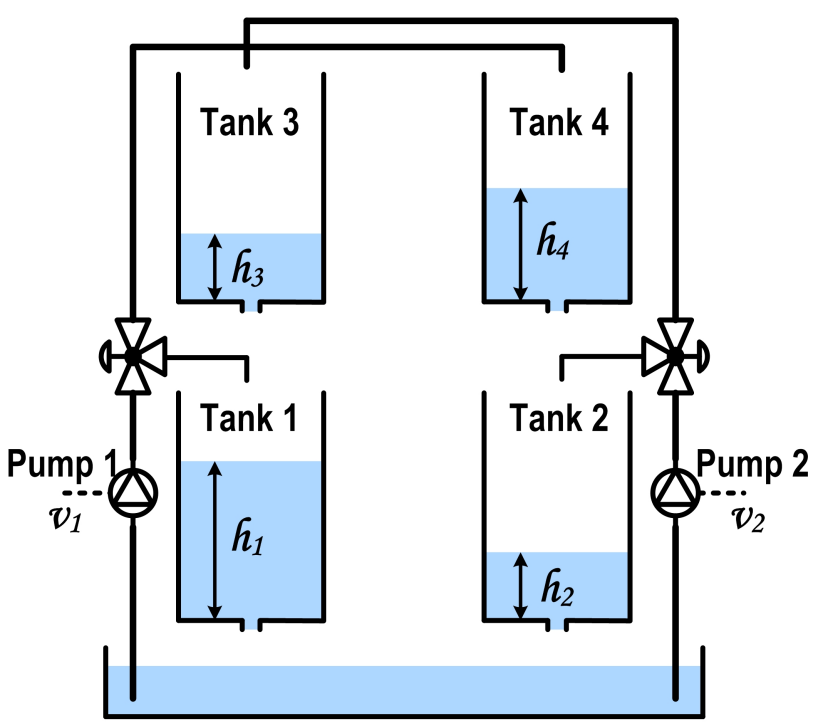

Figure 1: Quadruple-tank process

The by-pass valve $V_{1}$ derives a proportional part, $\gamma_{1}$, of the flow through pump $P_{1}$ to $T_{1}$ and the other part, $1-\gamma_{1}$, to $T_{4}$. Similarly, the flow through pump $P_{2}$ is diverted to $T_{2}$ and $T_{3}$ as a function of the value of $\gamma_{2}$.

To implement the proposed fault detection approach, the model of the four tank system should be expressed in discretetime as in (1). From the continuous-time non-linear equations presented in [13], the following non-linear discrete-time model can be obtained by using the Euler discretisation with a sampling time of $1 \mathrm{~s}$ :

$$
\begin{aligned}
x_{1 k+1} & =x_{1 k}-\xi_{1_{k}}+\frac{a_{3}}{A_{1}} \sqrt{2 g x_{3 k}}+\frac{\gamma_{1} k_{1}}{A_{1}} u_{1 k}, \\
x_{2 k+1} & =x_{2 k}-\xi_{2 k}+\frac{a_{4}}{A_{2}} \sqrt{2 g x_{4 k}}+\frac{\gamma_{2} k_{2}}{A_{2}} u_{2 k}, \\
x_{3 k+1} & =x_{3 k}-\xi_{3 k}+\frac{\left(1-\gamma_{2}\right) k_{2}}{A_{3}} u_{2 k}, \\
x_{4 k+1} & =x_{4 k}-\xi_{4 k}+\frac{\left(1-\gamma_{1}\right) k_{1}}{A_{4}} u_{1 k}, \\
y_{j_{k}} & =x_{j_{k}}, \quad \text { for } j \in\{1,2\},
\end{aligned}
$$

where:

- The state variables are the tank levels (i.e., $x_{i}=h_{i}$, $i=1, \ldots, 4)$.

- The measured variables are the levels in tanks $T_{1}$ and $T_{2}$ (i.e., $y_{1}=h_{1}$ and $y_{2}=h_{2}$ ).

- The measurement noise $\left(v_{i}, i=1, \ldots, 2\right)$ is assumed to be unknown but bounded and its bound is 0.1 .

- $\xi_{i}=\frac{a_{i}}{A_{i}} \sqrt{2 g x_{i k}}$, for $i \in\{1, \ldots, 4\}$, with $A_{1}=A_{3}=$ $28 \mathrm{~cm}^{2}, A_{2}=A_{4}=32 \mathrm{~cm}^{2}$ (cross-sections of tanks), $a_{1}=a_{3}=0.071 \mathrm{~cm}^{2}, a_{2}=a_{4}=0.057 \mathrm{~cm}^{2}$ (crosssections of outlet holes) and $g=981 \mathrm{~cm} / \mathrm{s}^{2}$ (gravity constant). 
- Parameters $k_{1}, k_{2}$ (pump constants), $\gamma_{1}$ and $\gamma_{2}$ are assumed to belong to the intervals $k_{1} \in[3.2250,3.2450]$, $k_{2} \in[3.2600,3.2800], \gamma_{1} \in[0.5150,0.6150]$ and $\gamma_{2} \in$ [0.4200,0.5200]. Uncertain parameters are considered time varying.

\subsection{Fault scenarios}

From the continuous-time non-linear equations presented in [13], a simulator has been implemented in Matlab/Simulink. In the simulations, three different types of faults have been considered to test the proposed fault detection approach:

- Faults in the pumps: $f_{P_{1}}$ and $f_{P_{2}}$ are faults in the pumps $P_{1}$ and $P_{2}$, respectively. In these faulty situations, pump gains $\left(k_{1}, k_{2}\right)$ are reduced to the $80 \%$ of their nominal value.

- Leaks in tanks: $f_{T_{1}}, f_{T_{2}}, f_{T_{3}}$ and $f_{T_{4}}$ are faults corresponding to leaks in tanks $T_{1}, T_{2}, T_{3}$ and $T_{4}$, respectively. These leaks are assumed to be produced by holes of size $a_{i} / 2$ at the bottom of the tanks (such that the outflow is lost).

- Faults in the level sensors: $f_{y_{1}}$ and $f_{y_{2}}$ are the faults in level sensors $y_{1}$ and $y_{2}$, respectively. These faults have been simulated by reducing the sensor gains to the $50 \%$ of their nominal value.

\subsection{Results}

The results corresponding to three of the previous fault scenarios are presented: a faultless scenario and two faulty situations. In all of them, results are obtained by using the Algorithm 2. As discussed in the background section, several approaches can be used to solve the ICSP enunciated in Algorithm 2, including Waltz's local filtering algorithm [23] and Hyvönen's tolerance propagation algorithm [10]. The first approach only ensures locally consistent solutions while the second can guarantee global consistent solutions but with high computational complexity. In this paper, the constraint satisfaction algorithm embedded in the ICSP tool Real Paver [9], that efficiently combines local and global consistency techniques, will be used to implement Algorithm 2.

\subsubsection{Scenario 1 (faultless)}

This scenario is used to test the set-membership state estimator in faultless situation. Results, obtained by using Algorithm 2, are presented in Figure 2. Top graphs of this figure show the lower and upper state estimate bounds for $h_{3}$ and $h_{4}$ respectively. Middle graphs show the temporal evolution of the measured levels $y_{1}$ and $y_{2}$ with each lower and upper state estimate bounds $\left(h_{1}, h_{2}\right)$. Finally, the bottom graph shows the value of the fault detection (FD) decision indicator. This indicator takes ' 0 ' value when no fault is detected and takes ' 1 ' value when a fault is detected. It can be noticed that in this case the FD decision indicator is not activated since it is a faultless scenario. Notice also that the predicted interval for the tank levels of $T_{1}$ and $T_{2}$ (i.e., $h_{1}$ and $h_{2}$ ) is very tight, while the interval for the tank levels of $T_{3}$ and $T_{4}$ (i.e., $h_{3}$ and $h_{4}$ ) is wider. The reason is due to the levels of $T_{1}$ and $T_{2}$ are measured and the only uncertainty is due to the measurement noise. On the other hand, $h_{3}$ and $h_{4}$ are estimated by using the model that is affected by parameter uncertainty. Although, they are indirectly refined by the level measurements $h_{1}$ and $h_{2}$.

\subsubsection{Scenario 2 (fault $f_{P_{1}}$ )}

In this scenario, a fault in pump $P_{1}$ is introduced at time $10 \mathrm{~min}$. Figure 3 shows the same variables as in Figure 2. It can be observed that when the fault $f_{P_{1}}$ appears, it is detected and the FD decision indicator changes from ' 0 ' to ' 1 '. Once the fault has been detected, the state estimation algorithm continues without correcting the estimation provided by the model using the measurements. This is a post-fault strategy that avoids the use of potentially faulty sensors. As a consequence, in both variables $y_{1}$ and $y_{2}$, the obtanied envelopes are wider, although the measurements still remain outside them.

\subsubsection{Scenario 3 (fault $f_{T_{3}}$ )}

Now, a leak in $\operatorname{tank} T_{3}$ is introduced at time $10 \mathrm{~min}$. Figure 4 shows the state estimate results. It can be noticed that when $f_{T_{3}}$ appears, the FD decision indicator changes form '0' to '1', what means that the fault is detected. The detection of this fault has a particular importance since it only appears in tank $T_{3}$, whose level is not measured. However, since it is estimated using the proposed state estimate approach, the fault could be detected.

Notice that, compared with the previous fault scenario, the inconsistency between the model output and the corresponding measurements only appears in the variable $y_{1}$. This information can be used for fault isolation.

\subsubsection{Scenario 4 (fault $f_{y_{1}}$ )}

Finally, a fault in the sensor that measures the level $h_{1}$ is introduced at time $10 \mathrm{~min}$. Figure 5 shows the state estimation results obtained using Algorithm 2. It can be noticed that when $f_{y_{1}}$ appears, the fault is indicated. As in the previous fault scenarios, once the inconsistency is detected using Algorithm 2 the state estimation algorithm continues without correcting the estate estimation by using the measurements. In this scenario, the measurements remain outside the envelopes just for some time instants. However, the FD decision is maintained assuming that once a fault appears it remains present.

\section{Application Example 2: Sewer network}

The second application example used to show the effectiveness of the proposed fault detection set-membership approach is based on the sewer network of Barcelona.

\subsection{System description}

The portion of sewer network of Barcelona considered in this paper is shown in Figure 6. This portion contain a real retention tank and two virtual tanks, which represent two network catchments [4]. The following non-linear model can be 

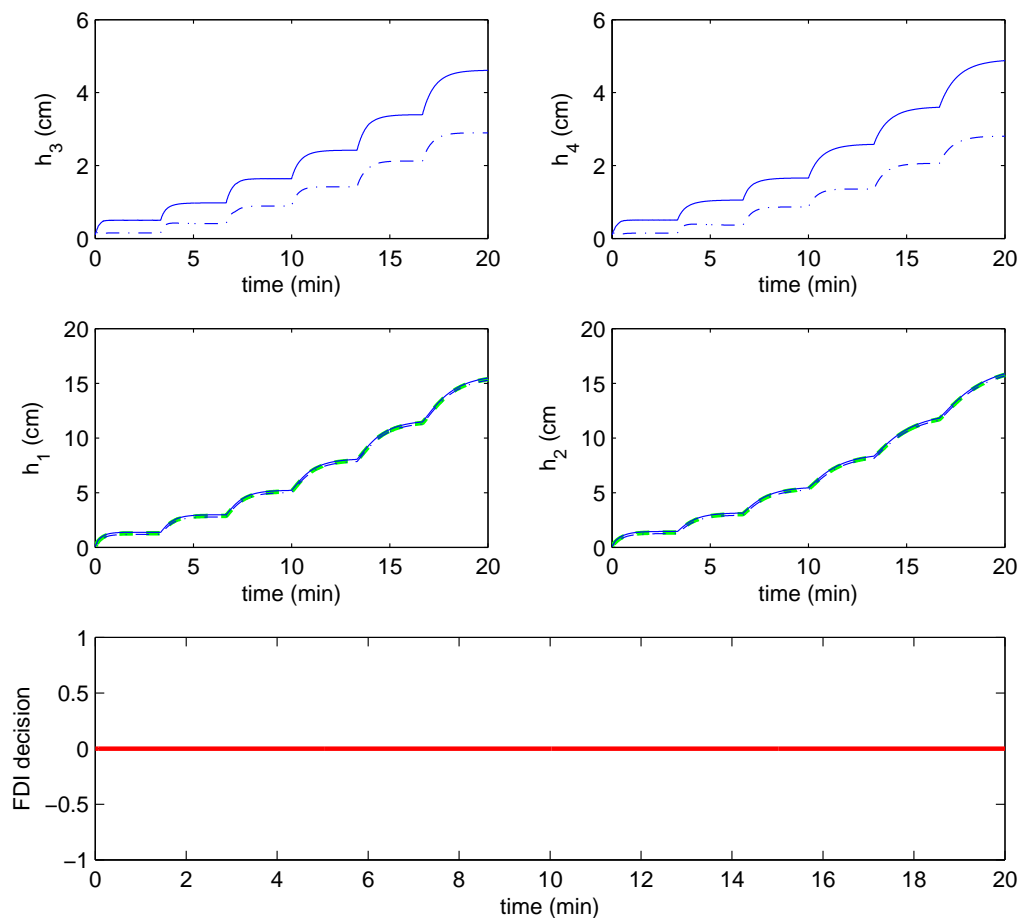

Figure 2: Results for the Quadruple-Tank process in the faultless scenario. Solid blue, upper bound estimated level. Dashed blue, lower bound estimated level. Dashed green, measured levels.
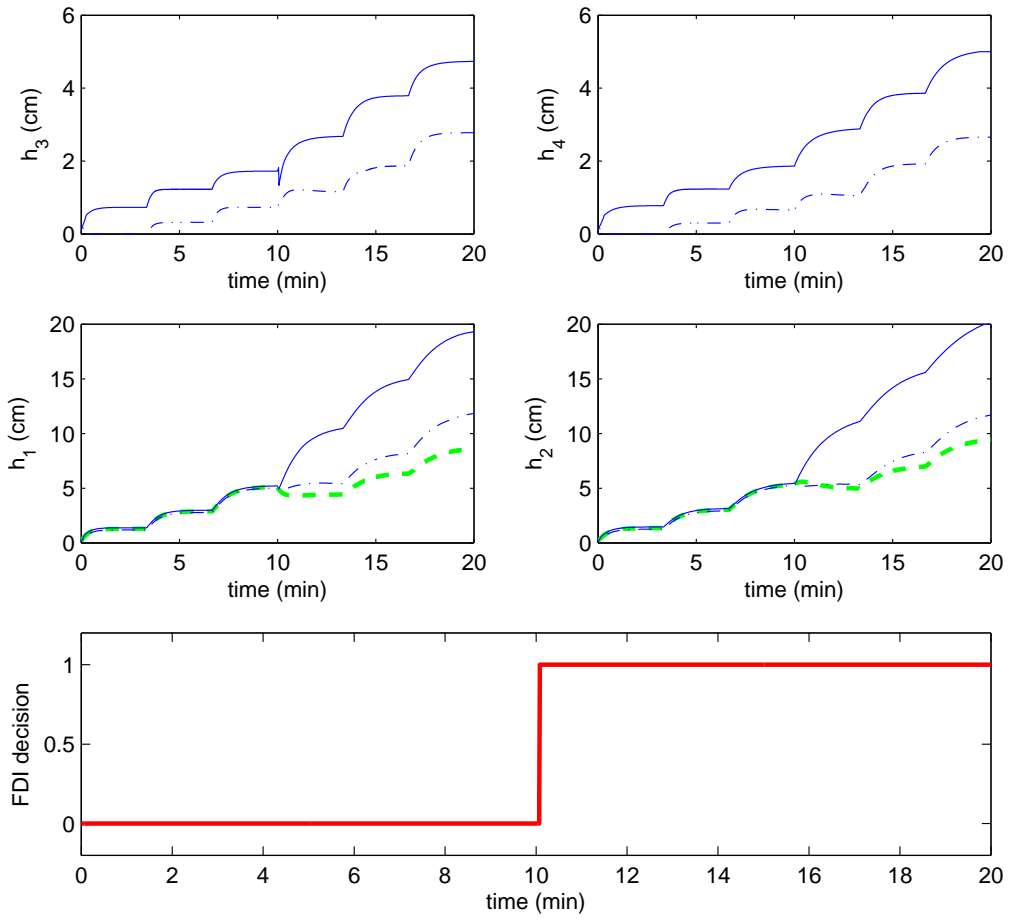

Figure 3: Results for the Quadruple-Tank process in the faulty scenario $f_{P_{1}}$. Solid blue, upper bound estimated level. Dashed blue, lower bound estimated level. Dashed green, measured levels. 

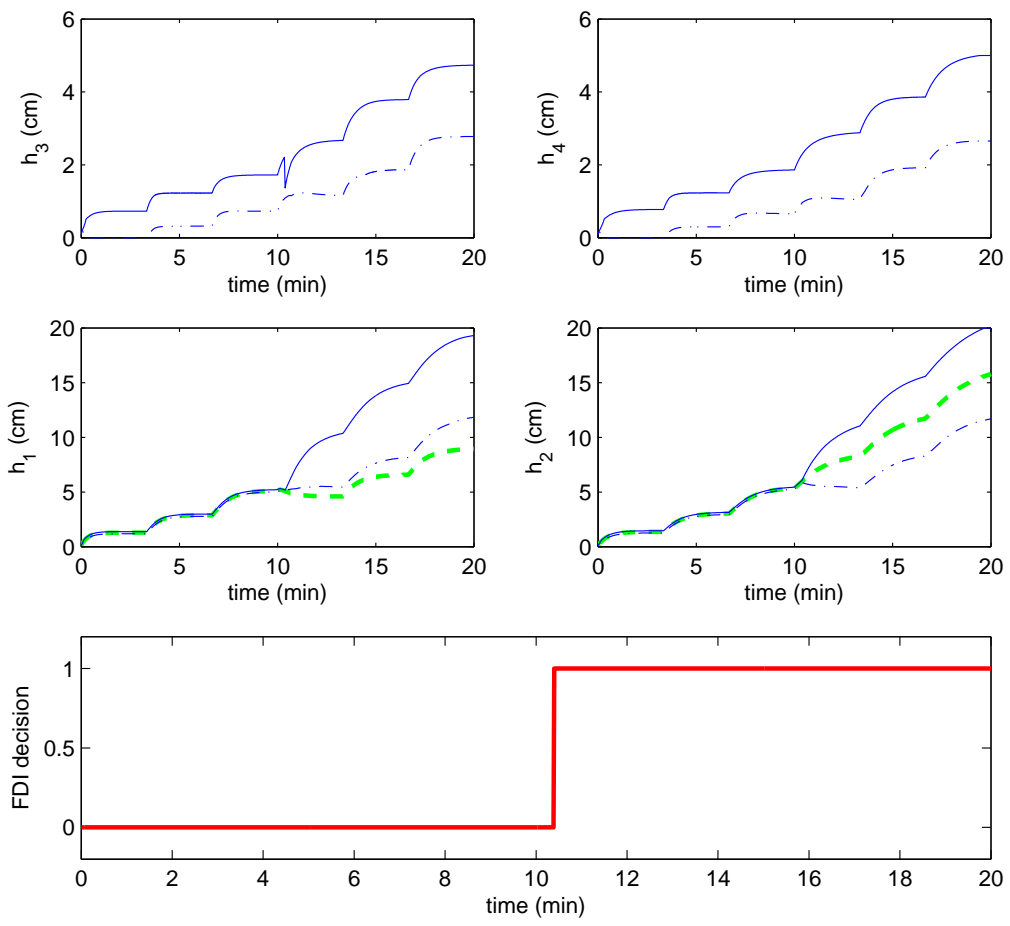

Figure 4: Results for the Quadruple-Tank process in the faulty scenario $f_{T_{3}}$. Solid blue, upper bound estimated level. Dashed blue, lower bound estimated level. Dashed green, measured levels.
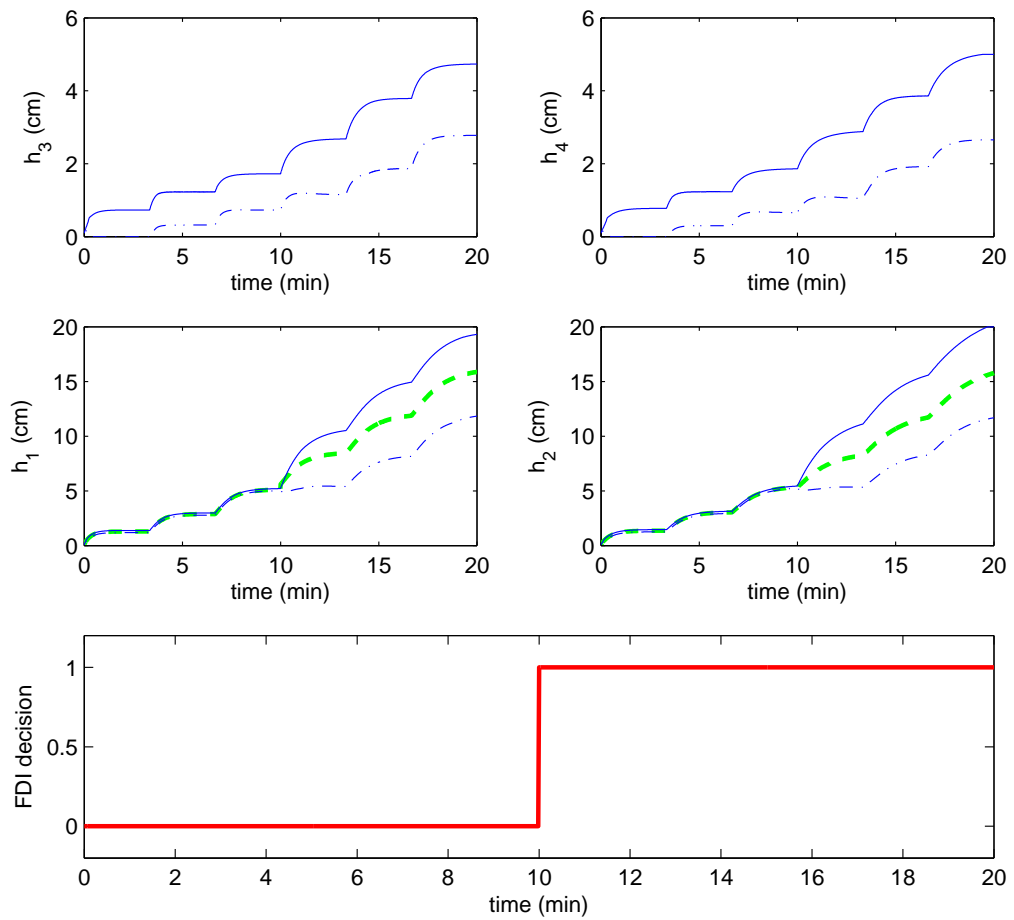

Figure 5: Results for the Quadruple-Tank process in the faulty scenario $f_{y_{1}}$. Solid blue, upper bound estimated level. Dashed blue, lower bound estimated level. Dashed green, measured levels. 
obtained by using the conservation of mass in tanks, assuming that the position of gates $C_{1}$ and $C_{2}$ are fixed to avoid the sewage flow through links $q_{1}$ and $q_{2}$, and that gate $C_{3}$ is completely open:

$$
\begin{aligned}
x_{1 k+1} & =x_{1 k}+T\left[u_{1 k}+u_{2 k}-\beta_{1} x_{1 k}\right], \\
x_{2 k+1} & =x_{2 k}+T\left[\beta_{1} x_{1 k}-\beta_{2} \sqrt{x_{2 k}}\right], \\
x_{3 k+1} & =x_{3 k}+T\left[\beta_{2} \sqrt{x_{2 k}}+u_{3 k}-\beta_{3} x_{3 k}\right], \\
y_{k} & =\beta_{2} \sqrt{x_{2 k}}+v_{k},
\end{aligned}
$$

where

- $x_{i}$ is the water volume in the $i$-th virtual/real tank $\mathrm{T}_{i}$.

- $u_{i}=d_{i}+w_{i}$ corresponds to the rain inflow measured employing rain gauges $\left(P_{19}, P_{16}\right.$ and $P_{20}$, according to Figure 6), being $u_{i}$ the real inflow and $w_{i}$ the measured noise such that $w_{i k} \in \mathbb{W}_{i}=[0,0.1]$.

- $y_{k}$ is the sewage level in the output sewer of the $\operatorname{tank} T_{2}$, measured by using the limnimeter $L_{47}$.

- $v_{k}$ is the associated noise defined by $\mathbb{V}_{i}=[-5,5]$.

- $\beta_{i}, i \in\{1,2,3\}$, corresponds with a proportional factor for the volume-flow conversion.

- The model parameters and associated uncertainty have been estimated from real data using the procedure described in [18]. The obtained values are $\beta_{1} \in[4.8 \times$ $\left.10^{-4}, 6.8 \times 10^{-4}\right], \beta_{2} \in\left[1.99 \times 10^{-2}, 2.01 \times 10^{-2}\right]$ and $\beta_{3} \in\left[9.9 \times 10^{-4}, 10.1 \times 10^{-4}\right]$.

- The system initial conditions that correspond with the initial sewer volume of the tanks are: $x_{10}=167 \mathrm{~m}^{3}$, $x_{20}=0 \mathrm{~m}^{3}$ and $x_{30}=333 \mathrm{~m}^{3}$.

- $T=300$ s is the sampling time.

\subsection{Results}

The presented results are obtained from data recorded by the telemetry system of Barcelona sewer network in a real rain scenario occurred on September 14, 1999. Algorithm 2 has been applied in faultless and faulty scenarios.

\subsubsection{Scenario 1 (faultless)}

Figure 7 shows the results obtained in a faultless scenario. Top graphs show the lower and upper state estimate bounds for the non-measured states $x_{1}$ and $x_{3}$. Middle graphs show the temporal evolution of the state $x_{2}$ estimated with the measured variable $y_{1}$ and the lower and upper state estimate bound. Finally, in the bottom graph, the value of the FD decision indicator is shown. From Figure 7, it can be noticed that the state estimation algorithm is working properly. It can also be seen that the fault indicator is not activated.

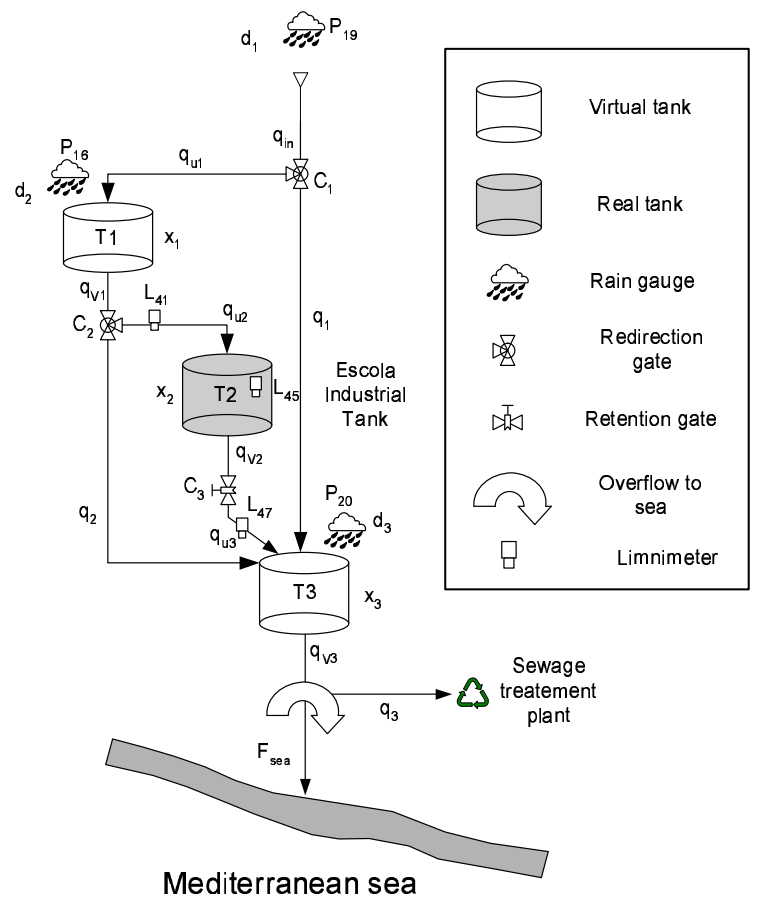

Figure 6: Case study: Portion of Barcelona's sewer network

\subsubsection{Scenario 2 (sensor fault)}

In this scenario, based on the same rain used in Scenario 1 , an additive sensor fault $f_{y}=100$ has been artificially introduced from $k=40$ (i.e., $200 \mathrm{~min}$ ). Figure 8 shows the same cases as in Figure 7. The FD decision indicator is activated just after the fault apparition. In this case, the estimated interval for state variable $x_{2}$ is empty, indicating the inconsistency between the model and the available measurements.

\section{Conclusions}

In this paper, robust fault detection using set-membership state estimation is presented. This state estimation is based on interval models that include parametric uncertainty. Additionally, process and measurement noise are considered to be unknown but bounded. First, the problem of set-membership state estimation implemented using constraints satisfaction techniques is presented. Then, the application of set-membership state estimation to fault detection is analysed. The main contribution of this paper involves the consideration of both modelling uncertainty and measurement noise in an unified way using the set-membership state estimation framework. Another important contribution is to show that constraints satisfaction may be an useful tool applied on fault detection.

Finally, two application examples have been used to show the effectiveness of the proposed approach: the well-known quadruple-tanks benchmark and a representative piece of Barcelona sewer network. The fault detection results obtained using the proposed approach show good performance in the considered scenarios. As further research, the algorithm is being extended to address the fault isolation, the second stage after fault detection in a fault diagnosis system. 

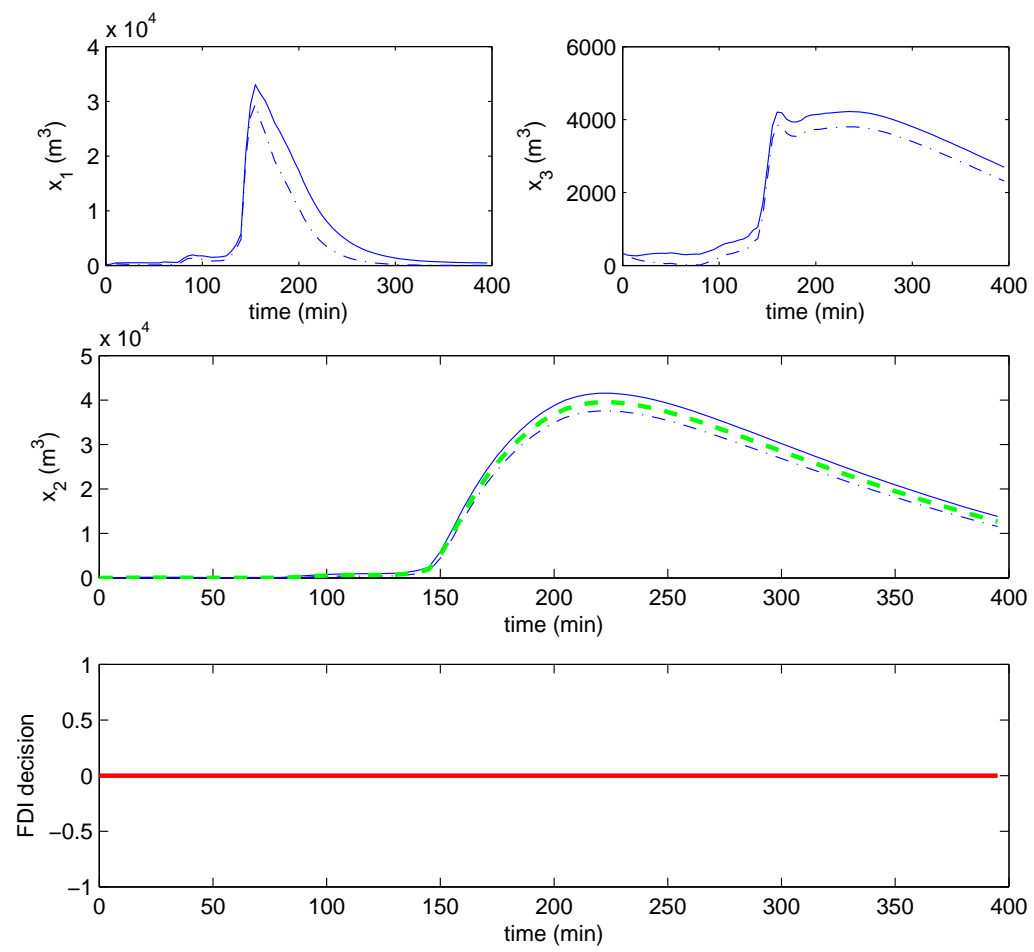

Figure 7: Results of the sewer network case study in the faultless scenario. Solid blue, upper bound estimated level. Dashed blue, lower bound estimated level. Dashed green, measured levels.

\section{Acknowledgments}

This work has been supported by the CICYT WATMAN (Ref. DPI2009-13744) and CICYT HYFA (Ref. DPI200801996) of the Spanish Science and Technology Ministry. The authors thank the support received from CLABSA in the second application presented in this paper.

\section{References}

[1] Angeli, D., Sontag, E., October 2003. Monotone control systems. IEEE Trans. Automatic Control 48 (10), 1684-1698.

[2] Basseville, M., Nikiforov, I., 1993. Detection of abrupt changes: theory and applications. Prentice Hall.

[3] Calafiore, G., 2001. A set-valued non-linear filter for robust localization. Proceedings of European Control Conference.

[4] Cembrano, G., Quevedo, J., Salamero, M., Puig, V., Figueras, J., Mart, J., 2004. Optimal control of urban drainage systems: a case study 12 (1), $1-9$.

[5] Chen, J., Patton, R., 1999. Robust Model-Based Fault Diagnosis for Dynamic Systems. Kluwer Academic Publishers.

[6] Chen, J., Patton, R., Zhang, H., 1996. Design of unknown input observers and robust fault detection filters. International Journal of Control 63 (1), 85-105.

[7] Cuguer, P., Puig, V., Saludes, J., Escobet, T., 2002. A class of uncertain linear interval models for which a set based robust simulation can be reduced to few pointwise simulations. Proceedings of IEEE Conference on Decision and Control 2, 1862-1863.

[8] Gertler, J., 1998. Fault Detection and Diagnosis in Engineering Systems. Marcel Dekker, New York.

[9] Granvilliers, L., Benhamou, F., 2006. Algorithm 852: Realpaver: an interval solver using constraint satisfaction techniques. ACM Trans. Math. Softw. 32 (1), 138-156.
[10] Hyvönen, E., 1992. Constraint reasoning based on interval arithmetic: The tolerance approach. Artificial Intelligence 58, 71-112.

[11] Jaulin, L., Kieffer, M., Braems, I., Walter, E., 2001. Guaranteed nonlinear estimation using constraint propagation on sets. International Journal of Control 74 (18), 1772-1782.

[12] Jaulin, L., Kieffer, M., Didrit, O., Walter, E., 2001. Applied Interval Analysis, with Examples in Parameter and State Estimation, Robust Control and Robotics. Springer-Verlag, London.

[13] Johansson, K., 2000. The Quadruple-Tank Process: A Multivariable Laboratory Process with an Adjustable Zero. IEEE Transactions on Control Systems Technology 8, 456-465.

[14] Kieffer, M., Jaulin, L., Walter, E., 2002. Guaranteed recursive non-linear state bounding using interval analysis. International Journal of Adaptive Control and Signal Processing 16 (3), 193-218.

[15] Maksarov, D., Norton, J., 1996. State bounding with ellipsoidal set description of the uncertainty. International Journal of Control 65 (5), 847 866.

[16] Milanese, M., Norton, J., Piet-Lahanier, H., Walter, E., 1996. Bounding Approaches to System Identification. Plenum Press.

[17] Ocampo-Martnez, C., Tornil, S., Puig, V., 2006. Robust fault detection using interval constraints satisfaction and set computations. In: Proceedings of IFAC SAFEPROCESS. Beijing (China).

[18] Ploix, S., Adrot, O., Ragot, J., 1999. Parameter uncertainty computation in static linear models. Proceedings of IEEE Conference on Decision and Control 2, 1916-1921.

[19] Puig, V., Quevedo, J., Escobet, T., De las Heras, S., 2002. Robust fault detection approaches using interval models. Proceedings of IFAC World Congress.

[20] Puig, V., Quevedo, J., Escobet, T., Morcego, B., Ocampo, C., 2004. Control tolerante a fallos (parte ii): Mecanismos de tolerancia y sistema supervisor. tutorial. Revista Iberoamericana de Automtica e Informtica Industrial 1 (2).

[21] Rinner, B., Weiss, U., 2004. Online monitoring by dynamically refining imprecise models. IEEE Trans. Syst., Man, Cybern. 34, 1811 - 1822. 

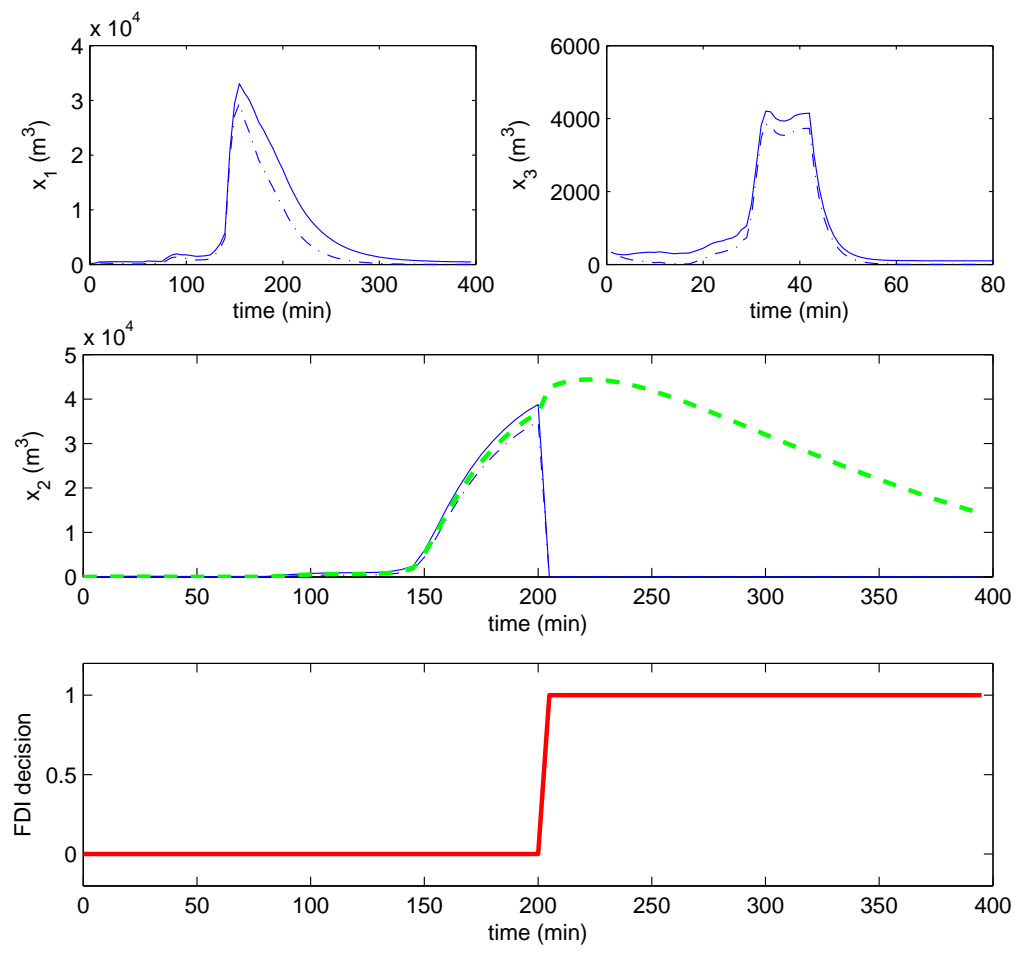

Figure 8: Results of the sewer network case study in a sensor-fault scenario. Solid blue, upper bound estimated level. Dashed blue, lower bound estimated level. Dashed green, measured levels.

[22] Shamma, J., 1997. Approximate set-value observer for nonlinear systems. IEEE Trans. Automatic Control 42 (5), 648 - 658.

[23] Waltz, D., 1975. Understanding line drawings of scenes with shadows. McGraw-Hill, New York.

[24] Witczak, M., Korbicz, J., Patton, R., 2002. A bounder-error approach to designing unknowninput observers. Proceedings of IFAC World Congress. 